\title{
Les humanités dans la formation et la professionnalisation des scientifiques
}

\section{Youssef Boughanmi}

\section{OpenEdition}

\section{Journals}

Édition électronique

URL : http://journals.openedition.org/trema/3633

DOI : $10.4000 /$ trema.3633

ISSN : 2107-0997

Éditeur

Faculté d'Éducation de l'université de Montpellier

Édition imprimée

Date de publication : 1 décembre 2017

Pagination : 1 - 4

ISBN : 979-10-96627-03-5

ISSN : 1167-315X

Référence électronique

Youssef Boughanmi, "Les humanités dans la formation et la professionnalisation des scientifiques », Tréma [En ligne], 47 | 2017, mis en ligne le 01 février 2018, consulté le 20 avril 2019. URL : http:// journals.openedition.org/trema/3633 ; DOI : 10.4000/trema.3633

Ce document a été généré automatiquement le 20 avril 2019.

Trema 


\title{
Les humanités dans la formation et la professionnalisation des scientifiques
}

\author{
Youssef Boughanmi
}

1 Les sciences humaines, en tant que champ de savoir relativement ancien, sont souvent liées aux actions, aux rapports et aux organisations de l'homme. Elles sont introduites par des arrêtés successifs (Bonah, 2013) dans les écoles d'ingénieurs, les instituts et les universités. Cette introduction a soulevé plusieurs problèmes institutionnels qui ont fait l'objet de maints débats sur l'utilité et les finalités de l'enseignement des sciences humaines à un public scientifique. Les méthodes de recherche spécifiques de ces disciplines diffèrent (Clément, 1999), en fonction de leurs objets, des autres sciences de la nature (matière, vivant). La double caractérisation des sciences humaines, spécificité et scientificité (Popper, 1973, Chalmers, 1987, Bachelard, 1947, Chazal, 2005), pose problème vu que ces sciences sont à la fois un patchwork de disciplines dont les objectifs sont très variés, et en même temps font appel à la modélisation et la quantification par leurs outils méthodologiques, utilisés aussi par les sciences de la nature. Plusieurs colloques, notamment celui de Besançon ${ }^{1} 2007$ où de Créteil ${ }^{2}$, 2013, ont consacré des symposiums pour discuter l'utilité et l'usage des sciences humaines dans la formation des scientifiques ou dans leurs cursus professionnels. Les finalités de la formation des scientifiques en sciences humaines sont plurielles et leurs mise en jeu est rendu possible grâce plusieurs domaines qui s'interfèrent: didactique, éthique, relations humaines, sociologie, histoire des sciences, psychologie...etc. Parfois science et humanité s'opposent, bien qu'elles soient liées par leurs différences, et les scientifiques se trouvent confrontés à des dilemmes qu'ils ne peuvent résoudre, selon les catégories traditionnelles de bienfaisance ou de malfaisance. L'enjeu du recours aux sciences humaines (Lemaître, 2001, 2011), notamment à l'éthique (Fabre, 2014), est de créer certaines finalités communes entre ces différentes disciplines. Cette question enrichit le débat sur le rôle des sciences humaines dans l'éducation à la citoyenneté, l'évolution des valeurs sociales et la réconciliation entre scientifiques et humanistes. Les papiers retenus pour ce numéro divulguent essentiellement deux pistes de recherche : les difficultés de l'enseignement des sciences 
humaines dans un environnement scientifique, mais surtout les spécificités des sciences humaines lorsqu'elles sont enseignées à des scientifiques appartenant à plusieurs domaines différents : ingénierie, médecine, biologie, formation des enseignants.

L'ensemble des textes, ici présentés, reprennent des problématiques spécifiques de chaque domaine des sciences humaines enseignés à un public scientifique varié. Chaque travail illumine l'apport des humanités (Citton, 2010 ; Dupuy, 2015) dans la formation et la professionnalisation des scientifiques. L'interaction entre les différents domaines n'est pas nécessairement disciplinaire, qui se manifeste par l'enseignement de plusieurs modules, mais fournit une réflexion sur les enjeux éducatifs, sociaux et institutionnels de ces enseignements. Chaque travail de recherche entreprend une certaine interdisciplinarité nourri d'une réflexion épistémologique sur les humanités et leurs spécificités dans un terrain réservé souvent aux sciences de la nature. A cet égard, un véritable dialogue interdisciplinaire opèrera des choix des contenus enseignés et leurs finalités.

\section{Humanités et ingénieurs}

Dans un volet, celui de l'éthique de l'ingénierie, et après avoir fait un état des lieux sur la formation à l'éthique des ingénieurs français, Didier mène une analyse comparative de l'évolution de l'éthique de l'ingénierie et celle des professionnels de la santé. En s'appuyant sur des comparaisons internationales, l'auteur montre la nécessité de donner à l'éthique de l'ingénierie sa place institutionnelle et réflexive, dans la formation et la professionnalité.

4 Roby, elle, a rembobiné l'histoire des SHS en écoles d'ingénieurs, elle a questionné l'actualité de ces humanités dans les institutions qui les a intégrées. L'auteur a mené une démonstration de l'évolution du statut et de la fonction de l'ingénieur qui a fortement changé. Le recours à l'histoire permet aussi, selon l'auteur, de faire évoluer la formation dans les écoles d'ingénieurs en intégrant des modules des sciences humaines en fonction des parcours.

\section{Humanités et enseignants}

Dupuy s'interroge sur l'implication de la culture humaniste dans la politique de la formation des enseignants. Après un retour sur les variations des humanités et leur influence sur le développement d'une culture humaniste chez les enseignants, elle mène une réflexion sur la réconciliation des humanités et leurs formations. Une nouvelle culture humaniste ne peut s'envisager qu'en élaborant un modèle de formation propre aux humanités culturelles basé sur l'interdisciplinarité. La réalité professionnelle l'oblige, des " sciences humaines appliquées à l'éducation » semblent répondre aux besoins de l'actuelle formation des enseignants.

6 Dans le cadre de la formation des enseignants, Abdelli traite l'apport de la didactique et de l'histoire des sciences et l'épistémologie à la formation des enseignants en éducation sexuelle dans ses dimensions culturelles, sociales et éducatives. Dans un cadre plus large celui de l'éducation de la citoyenneté, l'auteur montre comment l'éducation à la sexualité permet de développer des valeurs citoyennes communes et traite les relations entre groupes et individus. 
7 La place de l'histoire des sciences dans la formation des professeurs des écoles intrigue Egginger par son rôle pluridisciplinaire permettant aux étudiants de se doter une culture réflexive. L'auteur questionne la relation entre la construction historique du savoir et la construction personnelle des connaissances. Il interroge les difficultés des étudiants confrontés à l'apprentissage d'un savoir scientifique et montre le rôle que peut jouer l'histoire des sciences dans la motivation et le développement d'une certaine autonomie de construction du savoir chez les professeurs des écoles.

\section{Humanités et médecins}

Dans la cadre de la formation des médecins, Balez mène une étude sur la mise en situation des futurs médecins avant qu'ils exercent. Les situations sont construites en fonction de la réalité du terrain et portent sur la gestion d'un conflit, l'incertitude, l'agressivité d'un patient...etc. Il s'interroge alors sur la relation et la communication médicale en formant les médecins à la relation par la simulation.

La mise en œuvre de la formation a été réalisé à Brest auprès de quatre promotions de médecins et consiste un apport intéressant pour l'enseignement par la simulation en médecine.

Dans le même cadre de la formation des médecins, Aiguier vise à réfléchir à l'enseignement de l'éthique médicale comme compétence dans les formations médicinales. Il donne des ressources précieuses aux acteurs de la formation médicale et propose des pistes de réflexions aux médecins également. L'auteur montre que les critères de la professionnalisation en médecine ont changé, évoluent, et demandent d'émerger un nouveau besoin d'éthique. En termes pédagogiques, la professionnalisation de la médecine nécessite le passage d'une éthique axée sur les discours normatifs à propos des pratiques, à une capacité de « faire » de « l'éthique en situation ».

\section{Humanités et chercheurs biologistes}

11 Boughanmi et Saïd-Touhami s'interrogent sur la construction de la posture du chercheur par le biais d'un diagnostic des difficultés de l'élaboration d'un projet de recherche par des futurs biologistes. Les difficultés sont d'ordre méthodologique ou épistémologique et liées aussi à la spécificité de la biologie. Ces difficultés trouvent leur origine dans une représentation techniciste et utilitariste de la science. L'évolution des biologistes par l'avancement de leur projet dans cadre temporel et institutionnel, nourri de réflexions sur leurs procédures méthodologiques, forgera leurs postures de chercheurs. À l'heure actuelle, l'interaction entre humanités et sciences ne doit pas être seulement scientifique, elle devrait être une culture sociétale. 


\section{BIBLIOGRAPHIE}

Bachelard, (1947). Le rationalisme appliqué. Paris : PUF.

Bonah C. (2013). Expériences, comparaisons, mutualisations : l'exemple des enseignants de sciences humaines et sociales en santé. Colloque SHST, Créteil 2013.

Clément P. (1999). Les spécificités de la biologie et de son enseignement. Biologie géologie, 3.

Chalmers A.-F. (1987). Qu'est-ce que la science ? Éditions la découverte. Pour la traduction française.

Chazal G. (2005). La philosophie saisie par l'éducation. Rêver l'égalité, penser la culture. Tome 1, actes du colloque des 18 et 19, décembre 2003. CRDP, Bourgogne.

Citton, Y. (2010). L'avenir des humanités. Economie de la connaissance ou culture de l'interprétation? Paris, Editions de la Découverte

Dupuy, C. (2015). « Tentative d'épuisement de la culture humaniste en formation d'enseignants », Tréma [En ligne], n 43 | 2015, URL : http://trema.revues.org/3275.

Fabre, M. (2014). Minimalisme moral et maximalisme éthique chez John Dewey, Recherches en Education, Hors-Série n6, 71-80

Lemaître, D. (2011). Professionnalisation et modèles professionnels dans les grandes écoles françaises. Recherche \& Formation, 93-116.

Lemaître, D. (2001). Formation humaine dans les écoles d'ingénieurs : étude des conceptions contemporaines (dimensions anthropologique, éthique et culturelle). Doctorat NR, Nantes

Popper K. (1973). La logique de la découverte scientifique. Payot. (éd. originale : Londres, 1959).

\section{NOTES}

1. Colloque international « la scientificité des sciences humaines ». Besançon, 2007.

2. Colloque international «sciences humaines en sciences et techniques ». Créteil, 2013.

\section{AUTEUR}

\section{YOUSSEF BOUGHANMI}

Université de Bourgogne, Centre George Chevrier 